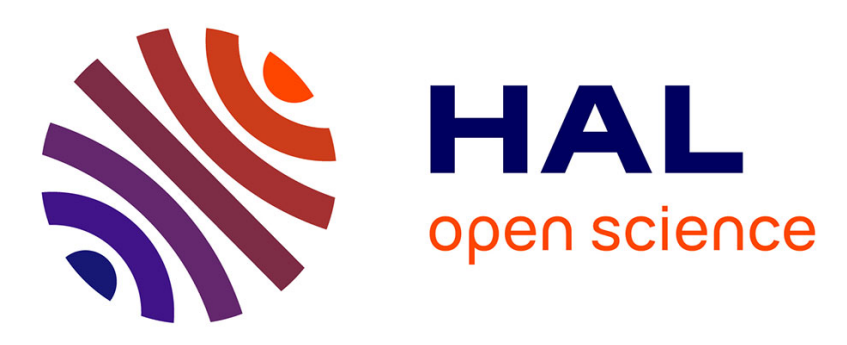

\title{
On the observer canonical form for Nonlinear Time-Delay Systems
}

Claudia Califano, Luis-Alejandro Marquez Martinez, Claude H. Moog

\section{To cite this version:}

Claudia Califano, Luis-Alejandro Marquez Martinez, Claude H. Moog. On the observer canonical form for Nonlinear Time-Delay Systems. 18th IFAC World Congress, Aug 2011, Milano, Italy. hal00584322

\section{HAL Id: hal-00584322 \\ https://hal.science/hal-00584322}

Submitted on 8 Apr 2011

HAL is a multi-disciplinary open access archive for the deposit and dissemination of scientific research documents, whether they are published or not. The documents may come from teaching and research institutions in France or abroad, or from public or private research centers.
L'archive ouverte pluridisciplinaire HAL, est destinée au dépôt et à la diffusion de documents scientifiques de niveau recherche, publiés ou non, émanant des établissements d'enseignement et de recherche français ou étrangers, des laboratoires publics ou privés. 


\title{
On the observer canonical form for Nonlinear Time-Delay Systems
}

\author{
C. Califano* L.A. Marquez-Martinez ${ }^{* *}$ C.H. Moog ${ }^{* * *}$ \\ * Dipartimento di Informatica e Sistemistica "Antonio Ruberti", \\ Sapienza Università di Roma, Via Ariosto 25, 00185 Rome, Italy. \\ ** CICESE-Depto. de electrónica y telecomunicaciones. \\ Carretera Ensenada-Tijuana No. 3918, Zona Playitas, 22860, \\ Ensenada B.C., Mexico. \\ *** IRCCyN, UMR C.N.R.S. 6597, 1 rue de la Noё, BP 92101, 44321 \\ Nantes Cedex 3, France.
}

\begin{abstract}
Necessary and sufficient geometric conditions for the equivalence of a nonlinear timedelay system with one output, under bicausal change of coordinates and output transformation, to a linear weakly observable time-delay system up to output injection are given. These conditions are derived through the use of the Extended Lie Bracket operator recently introduced in the literature for dealing with time-delay systems. The results presented show how this operator is useful in the analysis of this class of nonlinear systems.
\end{abstract}

Keywords: Nonlinear time-delay systems; differential geometry; observer canonical form; output injection.

\section{INTRODUCTION}

Conditions are derived under which a given nonlinear time-delay system is equivalent under bicausal change of coordinates and output transformation and up to output injection, to a linear weakly observable time-delay system. As it is the case for delay-free nonlinear systems, such an equivalence has the immediate benefit to transpose the problem of the reconstruction of the state of a nonlinear system, to a linear problem. In fact, it is possible to define an observer candidate such that the error between the state and its estimate is solution of a linear time-delay differential equation. Thus, the stability results known for such linear time-delay systems will be the tools to design an asymptotic observer.

In the delay-free case, this problem has been widely studied both for continuous-time and discrete-time systems (see for example Besancon et al. (1998) $\div$ Califano et al. (2009), Glumineau et al. (1996), Hammouri et al. (1996) $\div$ Huijberts (1999), Monaco et al. (2009), Xia et al. (1989), and the references therein). For time-delay systems the problem of the design of an observer has been addressed through several approaches. More precisely, in MarquezMartinez et al. (2002), sufficient conditions for the equivalence to the observer canonical form up to output injection are given through an algebraic approach while in MarquezMartinez et al. (2004) necessary and sufficient existence conditions are given still through the use of one forms; in Germani et al. (2002), the problem is solved through a chain observer by admitting delays on the output function only; finally in Anguelova et al. (2010) a new approach for the definition of the conditions under which a weakly observable system admits a unique state corresponding to a given input-output behavior is proposed.

In the present paper the analysis is performed following the geometric approach proposed in Califano et al. (2010) with respect to the linear equivalence problem and used in Califano et al. (2010b) for the analysis of time-delay systems. It is shown that such a geometric framework allows to define a constructive procedure for the computation of the desired bicausal change of coordinates. The results obtained not only may be generalized to the multi-output case as done in Califano et al. (2009) with reference to nonlinear discrete time systems, but enlighten the importance of the Extended Lie bracket operator for addressing control problems.

The paper is organized as follows. Some technical arguments concerning the geometric framework are given in Section 2. In Section 3 the main results are presented. An example displays the technical computations.

\section{PRELIMINARIES}

The class of nonlinear systems is considered, where the state and control variables are affected by possible multiple constant commensurate delays. The given dynamics can be modeled, without loss of generality through the equations

$$
\Sigma:\left\{\begin{aligned}
\dot{x}(t) & =F\left(\mathbf{x}_{[s]}\right)+\sum_{j=0}^{s} G_{j}\left(\mathbf{x}_{[s]}\right) u(t-j) \\
y & =H\left(\mathbf{x}_{[s]}\right)
\end{aligned}\right.
$$


where $\mathbf{x}_{[s]}=(x(t), \cdots x(t-s))$, with $x \in \mathbb{R}^{n}, u \in \mathbb{R}$, $y \in \mathbb{R}$. Throughout the paper we will denote by $\mathbf{x}_{[s]}(-p)=$ $(x(t-p), \cdots x(t-s-p))$. When no confusion is possible the subindex will be omitted so that $\mathbf{x}$ and $\mathbf{x}(-p)$ will stand for $\mathbf{x}_{[s]}$ and $\mathbf{x}_{[s]}(-p)$ respectively.

Furthermore, $\mathcal{K}$ denotes the field of meromorphic functions of a finite number of symbols in $\{x(t-i), u(t-i), \dot{u}(t-$ $\left.i), \ldots, u^{(k)}(t-i), i, k \in \mathbb{N}\right\} ; \mathcal{E}$ is the vector space spanned by the symbols $\left\{d x(t-i), d u(t-i), d \dot{u}(t-i), \ldots, d u^{(k)}(t-\right.$ $i), i, k \in \mathbb{N}\}$ over $\mathcal{K}$. The elements of this space are called 1-forms; $d$ is the standard differential operator that maps elements from $\mathcal{K}$ to $\mathcal{E}:=\operatorname{span}_{\mathcal{K}}\{d x(t-i), d u(t-$ $\left.i), d \dot{u}(t-i), \ldots, d u^{(k)}(t-i), i, k \in \mathbb{N}\right\} ; \delta$ represents the backward time-shift operator, that is for $a(\cdot), f(\cdot) \in$ $\mathcal{K}: \delta[a(t) d f(t)]=a(t-1) \delta d f(t)=a(t-1) d f(t-1) ;$ $\mathcal{K}(\delta]$ is the (left) ring of polynomials in $\delta$ with coefficients in $\mathcal{K}$. Every element of $\mathcal{K}(\delta]$ may be written as $\alpha(\delta]=\alpha_{0}(t)+\alpha_{1}(t) \delta+\cdots+\alpha_{r_{\alpha}}(t) \delta^{r_{\alpha}}, \quad \alpha_{i} \in \mathcal{K}$, where $r_{\alpha}=\operatorname{deg}(\alpha(\delta])$ the polynomial degree in $\delta$ of its argument. Addition and multiplication on this ring are defined by $\alpha(\delta]+\beta(\delta]=\sum_{i=0}^{\max \left\{r_{\alpha}, r_{\beta}\right\}}\left(\alpha_{i}(t)+\beta_{i}(t)\right) \delta^{i}$ and $\alpha(\delta] \beta(\delta]=\sum_{i=0}^{r_{\alpha}} \sum_{j=0}^{r_{\beta}} \alpha_{i}(t) \beta_{j}(t-i) \delta^{i+j}$; this ring is a non-commutative, Euclidean ring, Xia et al. (2002); $\Delta(\delta]=\operatorname{span}_{\mathcal{K}(\delta]}\left\{r_{1}, \ldots, r_{s}\right\}$, is the right module spanned over $\mathcal{K}(\delta]$ by the column elements $r_{1}, \ldots, r_{s} \in \mathcal{K}^{n}(\delta]$.

$\Sigma_{L}$, the differential form representation of $\Sigma$, is given by

$$
\Sigma_{L}:\left\{\begin{array}{l}
d \dot{x}=f\left(\mathbf{x}_{[s]}, \mathbf{u}_{[s]}, \delta\right) d x+g\left(\mathbf{x}_{[s]}, \delta\right) d u \\
d y=h\left(\mathbf{x}_{[s]}, \delta\right) d x
\end{array}\right.
$$

with

$$
\begin{array}{r}
f\left(\mathbf{x}_{[s]}, \mathbf{u}_{[s]}, \delta\right)=\sum_{i=0}^{s} \frac{\partial F\left(\mathbf{x}_{[s]}\right)}{\partial x(t-i)} \delta^{i}+\sum_{j=0}^{s} u(t-j) \sum_{i=0}^{s} \frac{\partial G_{j}\left(\mathbf{x}_{[s]}\right)}{\partial x(t-i)} \delta^{i} \\
g\left(\mathbf{x}_{[s]}, \delta\right)=\sum_{j=0}^{s} G_{j}\left(\mathbf{x}_{[s]}\right) \delta^{j}, h\left(\mathbf{x}_{[s]}, \delta\right)=\sum_{i=0}^{s} \frac{\partial H\left(\mathbf{x}_{[s]}\right)}{\partial x(t-i)} \delta^{i} .
\end{array}
$$

In this context we will thus consider bicausal change of coordinates as defined in Marquez-Martinez et al. (2002). We have

Definition 1. Consider the dynamics $\Sigma$ with state coordinates $x . z=\phi\left(\mathbf{x}_{[\alpha]}\right), \phi \in \mathcal{K}^{n}$ is a bicausal change of coordinates for $\Sigma$ if there exist an integer $\ell \in \mathbb{N}$ and a function $\phi^{-1}\left(\mathbf{z}_{[\ell]}\right) \in \mathcal{K}^{n}$ such that $x(t)=\phi^{-1}\left(\mathbf{z}_{[\ell]}\right)$.

Denoting by $d z=T\left(\mathbf{x}_{[\gamma]}, \delta\right) d x$ its associated differential form representation, one has that the following properties hold true:

P1) $T\left(\mathbf{x}_{[\gamma]}, \delta\right)=\sum_{i=0}^{\alpha} \frac{\partial \phi\left(\mathbf{x}_{[\alpha]}\right)}{\partial x(t-i)} \delta^{i}=\sum_{i=0}^{\alpha} T^{i}\left(\mathbf{x}_{[\gamma]}\right) \delta^{i}$, with $\gamma \leq \alpha$ is unimodular ${ }^{1}$

\footnotetext{
1 A polynomial matrix $T(\mathbf{x}, \delta)$ is called unimodular if it has a polynomial inverse.
}

P2) The inverse $T^{-1}(\mathbf{z}, \delta)$ has polynomial degree $\ell \leq(n-$

1) $\alpha$ and is given by $T^{-1}(\mathbf{z}, \delta)=\sum_{i=0}^{\ell} \frac{\partial \phi^{-1}\left(\mathbf{z}_{[\ell]}\right)}{\partial z(t-i)} \delta^{i}$.

Under the bicausal change of coordinates $z(t)=\phi\left(\mathbf{x}_{[\alpha]}\right)$, (2) is transformed into

$$
\begin{aligned}
d \dot{z}(t) & =\tilde{f}(\mathbf{z}, \mathbf{u}, \delta) d z+\tilde{g}(\mathbf{z}, \delta) d u \\
d y & =\tilde{h}(\mathbf{z}, \delta) d z
\end{aligned}
$$

with

$$
\begin{aligned}
& \tilde{f}(\mathbf{z}, \mathbf{u}, \delta)= \\
& \quad=\left[(T(\mathbf{x}, \delta) f(\mathbf{x}, \mathbf{u}, \delta)+\dot{T}(\mathbf{x}, \delta)) T^{-1}(\mathbf{x}, \delta)\right]_{\mid \mathbf{x}=\phi^{-1}(\mathbf{z})}(4) \\
& \tilde{g}(\mathbf{z}, \delta)=(T(\mathbf{x}, \delta) g(\mathbf{x}, \delta))_{\mid \mathbf{x}=\phi^{-1}(\mathbf{z})} \\
& \tilde{h}(\mathbf{z}, \delta)=\left(h(\mathbf{x}, \delta) T^{-1}(\mathbf{x}, \delta)\right)_{\mid \mathbf{x}=\phi^{-1}(\mathbf{z})}
\end{aligned}
$$

\subsection{The Extended Lie bracket and its properties}

The Extended Lie Bracket has been recently introduced for dealing with time-delay systems in Califano et al. (2010) where the linear equivalence problem has been addressed and solved. Let $r_{i}(\mathbf{x}, \delta)=\sum_{j=0}^{s} r_{i}^{j}(\mathbf{x}) \delta^{j}$, and set $r_{i}^{s+j}(\mathbf{x})=0$ for any $j>0$. We have:

Definition 2. Let $r_{i}(\mathbf{x}, \delta)=\sum_{j=0}^{s} r_{i}^{j}(\mathbf{x}) \delta^{j}, i=i, 2$. For any $k, l \geq 0$, the Extended Lie bracket $\left[r_{1}^{k}(\cdot), r_{2}^{l}(\cdot,)\right]_{E i}$, on $\mathcal{R}^{(i+\overline{1}) n}$, is recursively defined as

$$
\begin{aligned}
& {\left[r_{1}^{k}(\cdot), r_{2}^{l}(\cdot)\right]_{E 0}=} \\
& \sum_{i=0}^{k} \frac{\partial r_{2}^{l}(\mathbf{x})}{\partial x(t-i)} r_{1}^{k-i}(\mathbf{x}(-i))-\sum_{i=0}^{l} \frac{\partial r_{1}^{k}(\mathbf{x})}{\partial x(t-i)} r_{2}^{l-i}(\mathbf{x}(-i)) \\
& {\left[r_{1}^{k}(\cdot), r_{2}^{l}(\cdot)\right]_{E i}=} \\
& \sum_{j=0}^{\min (k, l, i)}\left(\left[r_{1}^{k-j}(\cdot), r_{2}^{l-j}(\cdot)\right]_{E 0}\right)_{\mid \mathbf{x}(-j)}^{T} \frac{\partial}{\partial x(t-j)}
\end{aligned}
$$

As in the delay free case it is convenient to introduce an Extended Lie derivative whose definition is given below and is slightly different from the one given in Oguchi (2007).

Definition 3. Given the function $\tau\left(\mathbf{x}_{[s]}\right)$ and the submodule element $r_{i}(\mathbf{x}, \delta)=\sum_{j=0}^{\bar{s}} r_{i}^{j}(\mathbf{x}) \delta^{j}$, the Extended Lie derivative $L_{r_{i}^{j}(\mathbf{x})} \tau\left(\mathbf{x}_{[s]}\right)$

$$
L_{r_{i}^{j}(\mathbf{x})} \tau\left(\mathbf{x}_{[s]}\right)=\sum_{l=0}^{j} \frac{\partial \tau\left(\mathbf{x}_{[s]}\right)}{\partial x(t-l)} r_{i}^{j-l}(\mathbf{x}(-l))
$$


Accordingly

$\left[r_{1}^{k}(\cdot), r_{2}^{l}(\cdot)\right]_{E i}=$
$=\sum_{j=0}^{\min (k, l, i)}\left(L_{r_{1}^{k-j}(\mathbf{x})} r_{2}^{l-j}(\mathbf{x})-L_{r_{2}^{l-j}(\mathbf{x})} r_{1}^{k-j}(\mathbf{x})\right)_{\mid \mathbf{x}(-j)}^{T} \frac{\partial}{\partial x(t-j)}$.

thus recovering the definitions of Lie derivative and Lie bracket in the delay free case.

From (1), consider now the module element

$$
\mathbf{F}(\mathbf{x}, \delta)=\sum_{j=0}^{n s} F^{j}(\mathbf{x}) \delta^{j}=\sum_{j=0}^{n s} F(\mathbf{x}) \delta^{j} .
$$

Thus, the $\mathrm{i}-$ th derivative of $\tau(\mathbf{x})$ computed for $\mathbf{u}=0$, is given by $\tau^{(i)}(\mathbf{x}, 0)=L_{F^{n s}(\mathbf{x})}^{i} \tau(\mathbf{x})$, for any $i$. It follows that the observability rank condition can be easily checked by considering the matrix $O(\mathrm{x}, \delta)$ defined by

$$
O(\mathbf{x}, \delta) d x=\left(\begin{array}{c}
d H(\mathbf{x}) \\
d L_{F^{n s}(\mathbf{x})} H(\mathbf{x}) \\
\vdots \\
d L_{F^{n s}(\mathbf{x})}^{n-1} H(\mathbf{x})
\end{array}\right)
$$

We have the following notions of observability.

Definition 4. System (1) is weakly observable locally around $\mathbf{u}=0$ if $O(\mathbf{x}, \delta)$ computed according to (10) has full rank $n$ locally around $\mathbf{x}_{0}$, is strongly observable if $O(\mathbf{x}, \delta)$ is unimodular.

We can now state the definition of nilpotent submodule, which generalizes that of nilpotent distribution and which is a key tool in many control problems.

Definition 5. A submodule $\Delta=\operatorname{span}\left\{r_{1}(\mathbf{x}, \delta), \cdots, r_{j}(\mathbf{x}, \delta)\right\}$ nonsingular locally around $\mathbf{x}^{0}$, with $r_{l}(\mathbf{x}, \delta)=\sum_{k=0}^{s} r_{l}^{k}(\mathbf{x}) \delta^{k}$ is nilpotent of order 0 if

$$
\left[r_{l}^{k}, r_{i}^{p}\right]_{E, 2 s}=0, \quad \forall k \leq p \in[0,2 s] .
$$

Definition 6. Consider the bicausal change of coordinates $z=\phi\left(\mathbf{x}_{[\alpha]}\right)$, with $d z=T(\mathbf{x}, \delta) d x$. In the new coordinates the submodule element $r(\mathbf{x}, \mathbf{u}, \delta)$ is transformed as

$$
\tilde{r}(\mathbf{z}, \mathbf{u}, \delta)=[T(\mathbf{x}, \delta) r(\mathbf{x}, \mathbf{u}, \delta)]_{\mid \mathbf{x}=\phi^{-1}(\mathbf{z})} .
$$

From (11), setting $T^{j}=0$ for $j>\alpha=\operatorname{deg}(T(\mathbf{x}, \delta))$ and $r^{j}=0$ for $j>\operatorname{deg}(r(\mathbf{x}, \mathbf{u}, \delta))$ one has

$$
\tilde{r}^{l}(\mathbf{z}, \mathbf{u})=\sum_{p=0}^{l}\left(T^{p}(\mathbf{x}) r^{l-p}(\mathbf{x}(-p), \mathbf{u}(-p))\right)_{\mid \mathbf{x}=\phi^{-1}(\mathbf{z})} .
$$

As a consequence one has the following result concerning the action of a bicausal change of coordinates on the extended Lie bracket (Califano et al. (2010)).

Lemma 1. Let $r_{i}(\mathbf{x}, \mathbf{u}, \delta)=\sum_{j=0}^{s} r_{i}^{j}(\mathbf{x}, \mathbf{u}) \delta^{j}, i=1,2$. Under the bicausal change of coordinates $z(t)=\phi\left(\mathbf{x}_{[\alpha]}\right)$, charac- terized by $d z=T(\mathbf{x}, \delta) d x, T(\mathbf{x}, \delta)=\sum_{j=0}^{\alpha} T^{j}(\mathbf{x}) \delta^{j}$ one has, for $k \leq l$,

$$
\begin{gathered}
{\left[\tilde{r}_{1}^{k}(\mathbf{z}, \mathbf{u}), \tilde{r}_{2}^{l}(\mathbf{z}, \mathbf{u})\right]_{E k}=} \\
=\left(\Gamma^{l-k}(\mathbf{x})\left[r_{1}^{k}(\mathbf{x}, \mathbf{u}), r_{2}^{l}(\mathbf{x}, \mathbf{u})\right]_{E k}\right)_{\mid \mathbf{x}=\phi^{-1}(\mathbf{z})}
\end{gathered}
$$

where, setting $T^{j}=0$ for $j>\alpha$,

$$
\Gamma^{l-k}(\mathbf{x})=\left(\begin{array}{ccc}
T^{0}(\mathbf{x}) & \cdots & T^{l-k}(\mathbf{x}) \\
& \ddots & \\
0 & & T^{0}(\mathbf{x}(-l+k))
\end{array}\right)
$$

Next theorem (Califano et al. (2010b)), enlightens the conditions under which a set of $n$ one-forms are exact and can be used to define a bicausal change of coordinates.

Theorem 1. Let $P_{n}(\mathbf{x}, \delta)=\left[r_{1}(\mathbf{x}, \delta), \cdots, r_{n}(\mathbf{x}, \delta)\right]$ be a full rank matrix, with $r_{i}=\sum_{j=0}^{s} r_{i}^{j}\left(\mathbf{x}_{[\beta]}\right) \delta^{j}$ which can be factorized as $P_{n}(\mathbf{x}, \delta)=T(\mathbf{x}, \delta) Q(\delta)$, with $T\left(\mathbf{x}_{[\beta]}, \delta\right)=$ $\sum_{i=0}^{\bar{s}} T^{i}\left(\mathbf{x}_{[\beta]}\right) \delta^{i}$ unimodular and such that $\beta \leq \bar{s}$. Then $T^{-1}(\mathbf{x}, \delta)$ defines a bicausal change of coordinates if and only if $\forall l, j \in[1, n] ; \quad$ and $\forall i \leq k \in[0,2 s]$

$$
\left[r_{j}^{i}(\mathbf{x}), r_{l}^{k}(\mathbf{x})\right]_{E i}=0,
$$

or equivalently $\forall l, j \in[1, n] ; \quad$ and $\quad \forall i \leq k \in[0,2 s]$

$$
\left[r_{j}^{i}(\mathbf{x}), r_{l}^{k}(\mathbf{x})\right]_{E 0}=0 .
$$

\section{MAIN RESULTS}

One major drawback encountered when dealing with nonlinear delay systems, is that they are infinite dimensional systems, so that the direct application of Frobenius Theorem is not possible. It is shown hereafter that thanks to the mathematical preliminaries in Section 2, it is possible to fully characterize those nonlinear time-delay systems which are equivalent under bicausal change of coordinates to the weakly observable linear canonical form up to nonlinear output injections. The constructive proof allows to compute the required change of coordinates.

Problem Statement (The Problem of the Equivalence to the Observer Form up to output injection): Given system (1) find, if possible, a bicausal change of coordinates $z=\phi\left(\mathbf{x}_{[\alpha]}\right)$, such that in the new coordinates the given system is weakly observable and reads

$$
\begin{aligned}
\dot{z}(t) & =\sum_{j=0}^{s} A_{j} z(t-j)+\psi\left(\mathbf{y}_{[s]}, \mathbf{u}_{[s]},\right) \\
y & =\sum_{j=0}^{s} C_{j} z(t-j)
\end{aligned}
$$

To solve the problem, search for a submodule element $r_{1}(\mathbf{x}, \delta)$ solution of 


$$
O(\mathbf{x}, \delta) r_{1}(\mathbf{x}, \delta)=\left(\begin{array}{llll}
0 & \cdots & 0 & a(\mathbf{x}, \delta)
\end{array}\right)^{T}
$$

with $a(\mathbf{x}, \delta)$ of minimal degree. If such an element exists, we can also compute iteratively, for $i=2, \cdots n+1$

$$
r_{i}(\mathbf{x}, \mathbf{u}, \delta)=f(\mathbf{x}, \mathbf{u}, \delta) r_{i-1}(\mathbf{x}, \mathbf{u}, \delta)-\dot{r}_{i-1}(\mathbf{x}, \mathbf{u}, \delta)
$$

Immediate consequences are the following:

Proposition 1. If $r_{1}(\mathbf{x}, \delta)$ satisfying (16) exists, then the observability matrix has full rank loc. around $\mathbf{u}=\mathbf{0}, \mathbf{x}^{0}$.

The proof is omitted for reasons of space and is based on the consideration that the matrix $O(\mathbf{x}, \delta)$ satisfies

$$
O(\mathbf{x}, \delta)\left(r_{1}(\mathbf{x}, 0, \delta) \cdots r_{n}(\mathbf{x}, 0, \delta)\right)=\left(\begin{array}{ccc}
0 & \cdots & a(\mathbf{x}, \delta) \\
\vdots & . \cdot & * \\
a(\mathbf{x}, \delta) & * & *
\end{array}\right)
$$

thus proving the result.

Proposition 2. Under any bicausal change of coordinates $z=\phi\left(\mathbf{x}_{[\alpha]}\right)$, with $d z=T(\mathbf{x}, \delta) d x$, the submodule elements $r_{i}(\mathbf{x}, \mathbf{u}, \delta)$ for $i \geq 1$ defined by (16), (17) are transformed according to (11).

Proof. We first have to show that (11) is satisfied for $i=1$. In fact we have that in the new coordinates $\tilde{H}(\mathbf{z})=$ $H(\phi(\mathbf{x}))$ and $\tilde{F}(\mathbf{z})=\left[\sum_{i=0}^{\alpha} \frac{\partial \phi(\mathbf{x})}{\partial x(t-i)} F(\mathbf{x}(-i))\right]_{\left.\right|_{x=\phi^{-1}(\mathbf{z})}}$. Iteratively, assume that $L_{\tilde{F}^{n \bar{s}}(\mathbf{z})}^{i-1} H(\mathbf{z})=\left(L_{F^{n s}(\mathbf{x})}^{i-1} H(\mathbf{x})\right)_{\left.\right|_{x=\phi^{-1}(\mathbf{z})}}$ which is true for $i=1$ then we have that,

$$
\begin{aligned}
& L_{\tilde{F}^{n \bar{s}}(\mathbf{z})}^{i} H(\mathbf{z})=L_{\tilde{F}\left({ }^{n \bar{s}} \mathbf{z}\right)}\left(L_{F^{n s}(\mathbf{x})}^{i-1} H(\mathbf{x})\right)_{\left.\right|_{x=\phi^{-1}(\mathbf{z})}} \\
& \quad=\sum_{j=0}^{n \bar{s}} \sum_{l=0}^{n s} \frac{\partial\left(L_{F^{n s}(\mathbf{x})}^{i-1} H(\mathbf{x})\right)}{\partial x(t-l)} \mid \frac{\partial \phi^{-1}(\mathbf{z}(-l))}{\partial z(t-j)} \tilde{F}(\mathbf{z}(-j)) \\
& =\left.\sum_{x=\phi^{-1}(\mathbf{z})}^{n s} \frac{\partial\left(L_{F(\mathbf{x})}^{i-1} H(\mathbf{x})\right)}{\partial x(t-l)} F(\mathbf{x}(-l))\right|_{x=\phi^{-1}(\mathbf{z})} \\
& =\left(L_{F^{n s}(\mathbf{x})}^{i} H(\mathbf{x})\right)_{\left.\right|_{x=\phi^{-1}(\mathbf{z})}}
\end{aligned}
$$

It follows that

$$
\begin{aligned}
& O(\mathbf{x}, \delta) r_{1}(\mathbf{x}, \delta)=\tilde{O}(\mathbf{z}, \delta)_{z=\phi(\mathbf{x})} T(\mathbf{x}, \delta) r_{1}(\mathbf{x}, \delta) \\
& =\left(\begin{array}{llll}
0 & \cdots & 0 & a(\mathbf{x}, \delta)
\end{array}\right)^{T}
\end{aligned}
$$

which implies that $\tilde{r}_{1}(\mathbf{z}, \delta)=\left[T(\mathbf{x}, \delta) r_{1}(\mathbf{x}, \delta)\right]_{x=\phi^{-1}(\mathbf{z})}$, due to the bicausality of the change of coordinates. Assume now that (11) is true for $i-1$. According to (4) we have

$$
\begin{aligned}
\tilde{r}_{i}(\mathbf{z}, \mathbf{u}, \delta) & =\tilde{f}(\mathbf{z}, \mathbf{u}, \delta) \tilde{r}_{i-1}(\mathbf{z}, \mathbf{u}, \delta)-\dot{\tilde{r}}_{i-1}(\mathbf{z}, \mathbf{u}, \delta) \\
& =\left[T(\mathbf{x}, \delta) r_{i}(\mathbf{x}, \mathbf{u}, \delta)\right]_{x=\phi^{-1}(\mathbf{z})}
\end{aligned}
$$

which ends the proof. $\triangleleft$

3.1 Linear equivalence under bicausal change of coordinates up to output injection

In the present section necessary and sufficient conditions for the equivalence to the weakly observable canonical form up to output injection under bicausal change of coordinates are given. The solution uses the submodule elements $r_{i}(\mathbf{x}, \mathbf{u}, \delta), i \in[1, n]$, computed according to (16) and (17). We have:

Theorem 2. System (1) is equivalent, under a bicausal change of coordinates, to a linear weakly observable delay system up to output injection if and only if there exist $r_{1}(\mathbf{x}, \delta)$ solution of (16) with $a(\mathbf{x}, \delta)=a(\delta)$ and two matrices $T^{-1}(\mathbf{x}, \delta)$ unimodular, and $Q(\delta)$ of full rank such that

a) $\mathcal{O}_{n}(\mathbf{x}, \mathbf{u})=\left(r_{1}(\mathbf{x}, \delta), \cdots r_{n}(\mathbf{x}, \mathbf{u}, \delta)\right)=T^{-1}(\mathbf{x}, \delta) Q(\delta)$

b) for $i, j \in[1, n]$ and $l \leq k \in[0,2 \bar{s}]$, the following relation are satisfied

$$
\left[r_{i}^{l}(\mathbf{x}), r_{j}^{k}(\mathbf{x})\right]_{E 0}=0 .
$$

c) $r_{n+1}(\mathbf{x}, \mathbf{u}, \delta)=T^{-1}(\mathbf{x}, \delta) q_{2}(\mathbf{y}, \mathbf{u}, \delta)$

Proof. Necessity. First consider the time-delay system (15) which is already in the canonical observer form up to output injection. Is is easily verified that for this system $r_{1}(\mathbf{x}, \delta)=r_{1}(\delta)$ solution of (16) with $a(\mathbf{x}, \delta)=a(\delta)$ exists and conditions a $) \div \mathrm{c}$ ) are satisfied since by construction for $i \in[1, n], r_{i}(\cdot, \delta)=r_{i}(\delta)$ and according to Proposition 1 they are linearly independent. Furthermore

$$
\begin{aligned}
r_{n+1} & =f(\mathbf{z}, \mathbf{u}, \delta) r_{n}(\delta)=A(\delta) r_{n}(\delta)+\eta(\mathbf{y}, \mathbf{u}, \delta) \\
& =q_{2}(\mathbf{y}, \mathbf{u}, \delta) .
\end{aligned}
$$

From Proposition 2 and Lemma 1 , conditions a) $\div$ b) are invariant under a bicausal change of coordinates. Condition c) is an immediate consequence of (18) and Proposition 2.

Sufficiency. Assume that the conditions are satisfied. According to Theorem 1 , since $T^{-1}(\mathbf{x}, \delta)$ is unimodular and b) is satisfied, we can consider the bicausal change of coordinates $\mathbf{z}=\phi\left(\mathbf{x}_{[\alpha]}\right)$ such that $d z=T(\mathbf{x}, \delta) d x$. According to a) the $r_{i}(\mathbf{x}, \mathbf{u}, \delta)$ 's do not depend on the control variable and thus in the new coordinates, due to Proposition 2,

$$
\begin{gathered}
\left(\tilde{r}_{1}(\mathbf{z}, \delta), \cdots \tilde{r}_{n}(\mathbf{z}, \delta)\right)= \\
=\left[T(\mathbf{x}, \delta)\left(r_{1}(\mathbf{x}, \delta), \cdots r_{n}(\mathbf{x}, \delta)\right)\right]_{\mathbf{x}=\phi^{-1}(\mathbf{z})}=Q(\delta)
\end{gathered}
$$

It follows that the output $H\left(\mathbf{z}_{[s]}\right)$ is such that

$$
\begin{aligned}
& \sum_{i=0}^{s} \frac{\partial H}{\partial z(t-i)} \delta^{i}\left(\tilde{r}_{1}(\mathbf{z}, \delta), \cdots \tilde{r}_{n}(\mathbf{z}, \delta)\right)= \\
& h(\mathbf{z}, \delta)\left(\tilde{r}_{1}(\mathbf{z}, \delta), \cdots \tilde{r}_{n}(\mathbf{z}, \delta)\right) \\
& =\left(h(\mathbf{x}, \delta) T^{-1}(\mathbf{x}, \delta)\right)_{\mathbf{x}=\phi^{-1}(\mathbf{z})}\left(\tilde{r}_{1}(\mathbf{z}, \delta), \cdots \tilde{r}_{n}(\mathbf{z}, \delta)\right) \\
& =\left(\begin{array}{lllll}
0 & 0 & \cdots & 0 & a(\delta)
\end{array}\right)
\end{aligned}
$$


Multiplying on the right by $\operatorname{adj}[Q(\delta)]$, one gets

$$
\begin{aligned}
h(\mathbf{z}, \delta)[\operatorname{det}(Q(\delta)] & =a(\delta)\left(\begin{array}{llll}
\bar{q}_{n 1} & \bar{q}_{n 2} & \cdots & \bar{q}_{n n}
\end{array}\right) \\
& =C(\delta)[\operatorname{det}(Q(\delta)]
\end{aligned}
$$

where $\left(\begin{array}{llll}\bar{q}_{n 1} & \bar{q}_{n 2} & \cdots & \bar{q}_{n n}\end{array}\right)$ denotes the last row of the matrix $\operatorname{adj}[Q(\delta)]$. Relation (19) shows in fact the linearity of the output function in the new coordinates, due to the identity of polynomials. As for the forced dynamics, similarly, we have that due to $\mathrm{c}$ ),

$$
\begin{gathered}
\tilde{f}(\mathbf{z}, \mathbf{u}, \delta)\left(\tilde{r}_{1}(\delta), \cdots \tilde{r}_{n}(\delta)\right)= \\
\left(\tilde{r}_{2}(\mathbf{z}, \delta), \cdots, \tilde{r}_{n}(\mathbf{z}, \delta), \tilde{r}_{n+1}(\mathbf{z}, \mathbf{u}, \delta)\right)=\left[Q_{1}(\delta), q_{2}(\mathbf{y}, \mathbf{u}, \delta)\right] .
\end{gathered}
$$

Multiplying again on the right by $\operatorname{adj}[Q(\delta)] a(\delta)$, one gets

$$
\begin{gathered}
\tilde{f}(\mathbf{z}, \mathbf{u}, \delta)[\operatorname{det}(Q(\delta)] a(\delta)= \\
=Q_{2}(\delta)+q_{2}(\mathbf{y}, \mathbf{u}, \delta)\left(\bar{q}_{n 1} \bar{q}_{n 2} \cdots \bar{q}_{n n}\right) a(\delta) \\
=A(\delta)\left[\operatorname{det}(Q(\delta)] a(\delta)+\bar{q}_{2}(\mathbf{y}, \mathbf{u}, \delta) C(\delta)[\operatorname{det}(Q(\delta)] a(\delta)\right.
\end{gathered}
$$

that is, the system in the $\mathrm{z}$-coordinates reads (15) since $\tilde{f}(\mathbf{z}, \mathbf{u}, \delta)=A(\delta)+\bar{q}_{2}(\mathbf{y}, \mathbf{u}, \delta) C(\delta) . \triangleleft$

An immediate consequence is the following.

Corollary 1. System (1) is equivalent, under a bicausal change of coordinates, to a linear weakly observable delay system, if and only if there exist two matrices $T^{-1}(\mathbf{x}, \delta)$ unimodular, and $Q(\delta)$ of full rank such that conditions a) $\div$ c) of Theorem 2 are satisfied and

$$
\text { d') } r_{n+1}(\mathbf{x}, \mathbf{u}, \delta) \in \operatorname{span}_{\mathcal{K}(\delta]}\left\{r_{1}(\mathbf{x}, \delta), \cdots, r_{n}(\mathbf{x}, \delta)\right\}
$$

Remark. Under appropriate assumptions, necessary and sufficient existence conditions equivalent to Theorem 2 were given in Marquez-Martinez et al. (2004). However the conditions given in Theorem 2 are constructive, since the module elements $r_{i}(\mathbf{x}, \delta)$ characterize the change of coordinates. In particular if the system is strongly observable, then $\mathcal{O}_{n}(\mathbf{x}, \delta)$ is exactly the inverse of the bicausal change of coordinates differential form representation. $\triangleleft$

\subsection{An Example}

Consider the following nonlinear delay system

$$
\begin{aligned}
\dot{x}_{1}(t)= & x_{2}(t-1)+2 x_{2}(t)+\left[x_{1}(t)-x_{2}^{2}(t-1)\right]^{2} \\
& +2 x_{2}(t-1)\left(\left[x_{1}(t-2)-x_{2}^{2}(t-3)\right]^{2}+u(t-1)\right) \\
\dot{x}_{2}(t)= & {\left[x_{1}(t-1)-x_{2}^{2}(t-2)\right)^{2}+u(t) } \\
y(t)= & x_{1}(t)-x_{2}^{2}(t-1)
\end{aligned}
$$

We have that $L_{F} H(\mathbf{x})=x_{2}(t-1)+2 x_{2}(t)+\left[x_{1}(t)-\right.$ $\left.x_{2}^{2}(t-1)\right]^{2}$, so that, setting $c(\mathbf{x})=2\left(x_{1}(t)-x_{2}^{2}(t-1)\right)$, the observability matrix is

$$
O=\left(\begin{array}{cc}
1 & -2 x_{2}(t-1) \delta \\
c(\mathbf{x}) & \left.2+\delta-c(\mathbf{x}) 2 x_{2}(t-1) \delta\right)
\end{array}\right)
$$

which is of full rank but not unimodular. Let us thus compute, if it exists, $r_{1}(\mathbf{x}, \delta)$ solution of (16), that is s.t.

$$
\left(\begin{array}{cc}
1 & -2 x_{2}(t-1) \delta \\
c(\mathbf{x}) & \left.2+\delta-c(\mathbf{x}) 2 x_{2}(t-1) \delta\right)
\end{array}\right) r_{1}(\mathbf{x}, \delta)=\left(\begin{array}{c}
0 \\
a(\delta)
\end{array}\right)
$$

Standard computations show that

$$
r_{1}(\mathbf{x}, \delta)=\left(\begin{array}{c}
2 x_{2}(t-1) \delta \\
1
\end{array}\right)=\left(\begin{array}{l}
0 \\
1
\end{array}\right)+\left(\begin{array}{c}
2 x_{2}(t-1) \\
0
\end{array}\right) \delta
$$

To check the conditions of Theorem 2, we have now to compute $r_{2}(\mathbf{x}, \mathbf{u}, \delta)$, and $r_{3}(\mathbf{x}, \mathbf{u}, \delta)$. We have that

$$
\begin{aligned}
& r_{2}(\mathbf{x}, \mathbf{u}, \delta)=\left(\begin{array}{c}
\delta+2 \\
0
\end{array}\right)=\left(\begin{array}{l}
2 \\
0
\end{array}\right)+\left(\begin{array}{l}
1 \\
0
\end{array}\right) \delta \\
& r_{3}(\mathbf{x}, \mathbf{u}, \delta)=\left(\begin{array}{cc}
2 x_{2}(t-1) \delta & 1 \\
1 & 0
\end{array}\right)\left(\begin{array}{c}
2 c(\mathbf{x}(-1)) \delta \\
2 c(\mathbf{x})
\end{array}\right)
\end{aligned}
$$

Condition a) of Theorem 2 is thus satisfied. As for condition b) and d) we readily have that

$$
\begin{aligned}
\mathcal{O}_{n} & =\left(r_{1}(\mathbf{x}, \delta), r_{2}(\mathbf{x}, \delta)\right)=\left(\begin{array}{cc}
2 x_{2}(t-1) \delta & \delta+2 \\
1 & 0
\end{array}\right) \\
& =\left(\begin{array}{cc}
2 x_{2}(t-1) \delta & 1 \\
1 & 0
\end{array}\right)\left(\begin{array}{cc}
1 & 0 \\
0 & \delta+2
\end{array}\right)
\end{aligned}
$$

so that it is easily verified that they are satisfied with $T^{-1}(\mathbf{x}, \delta)=\left(\begin{array}{cc}2 x_{2}(t-1) \delta & 1 \\ 1 & 0\end{array}\right), Q(\delta)=\left(\begin{array}{cc}1 & 0 \\ 0 & \delta+2\end{array}\right)$, and $q_{2}(\mathbf{y}, \mathbf{u}, \delta)=\left(\begin{array}{c}2 y(t-1) \delta \\ 2 y(t)\end{array}\right)$

Finally we have to test condition c) that is the nilpotency of $\Delta=\operatorname{span}_{\mathcal{K}(\delta]}\left\{r_{1}(\mathbf{x}, \delta), r_{2}(\mathbf{x}, \delta)\right\}$, where the maximum delay $s=1$. The nilpotency can be easily verified by referring to the following matrix constructed starting form $r_{1}(\mathbf{x}, \delta)$ and $r_{2}(\mathbf{x}, \delta)$

$\left(\begin{array}{cccccc}R_{1}^{0} & R_{2}^{0} & R_{1}^{1} & R_{2}^{1} & R_{1}^{2} & R_{2}^{2} \\ \hline 0 & 2 & 2 x_{2}(t-1) & 1 & 0 & \\ 1 & 0 & 0 & 0 & 0 & \\ & & 0 & 2 & 2 x_{2}(t-2) & 1 \\ & & 1 & 0 & 0 & 0 \\ & & 0 & 0 & 0 & 2 \\ & & 0 & 0 & 1 & 0\end{array}\right)$

which shows that $\left[r_{1}^{0}, r_{2}^{0}\right]_{E 0}=\left[r_{1}^{0}, r_{1}^{1}\right]_{E 0}=\left[r_{1}^{0}, r_{2}^{1}\right]_{E 0}=$ $\left[r_{1}^{0}, r_{1}^{2}\right]_{E 0}=\left[r_{1}^{0}, r_{2}^{2}\right]_{E 0}=\left[r_{1}^{1}, r_{1}^{2}\right]_{E 0}=0$ as well as $\left[r_{2}^{0}, r_{2}^{2}\right]_{E 0}=\left[r_{2}^{1}, r_{2}^{2}\right]_{E 0}=\left[r_{1}^{2}, r_{2}^{2}\right]_{E 0}=0$. It follows that the bicausal change of coordinates $z_{1}(t)=x_{2}(t), z_{2}(t)=$ $x_{1}(t)-x_{2}^{2}(t-1)$ transforms the given dynamics into 


$$
\begin{aligned}
\dot{z}_{1}(t) & =y^{2}(t-1)+u(t) \\
\dot{z}_{2}(t) & =2 z_{1}(t)+z_{1}(t-1)+y^{2}(t) \\
y(t) & =z_{2}(t)
\end{aligned}
$$

\section{REFERENCES}

M. Anguelova \& B. Wennberg, On analytic and algebraic observability of nonlinear delay systems, Autom., 46, pp. 682-686, 2010.

P.S. Banks, Nonlinear delay systems, Lie algebras and Lyapunov transformations, J. of Math. Control and Info., 19, pp.59-72, 2002.

G. Besançon, H. Hammouri \& S. Benamor State equivalence of discrete-time nonlinear control systems to state affine form up to input/output injection, SCL, 33, pp. 1-10, 1998.

D. Bestle \& M. Zeitz, Canonical form observer design for non-linear time-variable systems, IJC, pp.419-431, 1983.

C. Califano, S. Monaco \& D. Normand-Cyrot, Canonical observer forms for multi-output systems up to coordinate and output transformations in discrete time, Autom., 45, pp. 2483-2490, 2009.

C. Califano, L.A. Marquez-Martinez \& C.H. Moog, On Linear Equivalence for Time-Delay Systems, ACC10, Baltimore, USA, June 2010.

C. Califano, L.A. Marquez-Martinez \& C.H. Moog, Extended Lie Brackets for Nonlinear Time-Delay Systems, preprint, 2010.

G. Conte, C. H. Moog, \& A.M. Perdon, Algebraic methods for nonlinear control systems, 242, 2nd ed. SpringerVerlag, 2007.

F.R. Gantmacher, Théorie des matrices, Series: Collection universitaire de mathématiques, Ed. Dunod, Paris, 1, 1966.

A. Germani, C. Manes \& P. Pepe, A new approach to state observation of Nonlinear Systems with delayed output, WSEAS TS ,1, pp.96-101, 2002.

A. Glumineau, C.H. Moog, \& F. Plestan, New algebrogeometric conditions for the linearization by inputoutput injection, IEEE TAC, 41, pp.598-603, 1996.

M. Halas, An Algebraic Framework Generalizing the Concept of Transfer Functions to Nonlinear Systems,Autom., 44, pp.1181-1190, 2008.

H. Hammouri \& M. Kinnaert, A new procedure for timevarying linearization up to output injection, SCL, 28, pp.151-157, 1996.

H.J.C. Huijberts, On existence of extended observers for nonlinear discrete-time systems, Lect. Notes in Cont. and Info. Sci., 244, pp.73-92, Springer-Verlag, 1999.

A. Isidori, Nonlinear Control Systems, Springer-Verlag, 3rd Ed., 1995.

E.B. Lee, \& A. Olbrot, Observability and related structural results for linear hereditary systems, IJC, 34, pp., 1981

I. Karafyllis \& C. Kravaris, On the observer problem for discrete-time control systems, IEEE TAC, 52, pp.12-25, 2007.

N. Kazantzis \& C. Kravaris, Nonlinear Observer Design using Lyapunov's auxiliary theorem, SCL, 34, pp.241247, 1998.

A.J. Krener \& A. Isidori, Linearization by output injection and nonlinear Observers, SCL, 3, pp.47-52, 1983.
A.J. Krener \& W. Respondek, Nonlinear Observers with linearizable error dynamics, SIAM J.Contr. Opt., 23, pp. 197-216, 1985.

A.J. Krener \& M. Xiao, Observers for linearly unobservable nonlinear systems, SCL, 46, pp.281-288, 2002

W. Lin \& C.I. Byrnes, Remarks on Linearization of discrete-time autonomous systems and nonlinear observer design, SCL, 25, pp. 31-40, 1995.

L.A. Marquez-Martinez, C.H. Moog \& M. Velasco-Villa, Observability and observers for nonlinear systems with time delay, Kyber., 38, pp.445-456, 2002.

L.A. Marquez-Martinez \& C.H. Moog, InputOutput Feedback Linearization of Time-Delay Systems, IEEE TAC, 49, pp.781-786, 2004.

W. Michiels \& S.-I. Niculescu, Stability and Stabilization of Time-Delay Systems. An Eigenvalue-Based Approach, Advances in Design and Control, 12, SIAM: Philadelphia, 2007

S. Monaco \& D. Normand-Cyrot, Linearization by Output Injection under Approximate Sampling, EJC, 15, pp.205-217,2009.

P.E. Moraal \& J.W. Grizzle, Observer design for nonlinear systems with discrete-time measurement, IEEE TAC, 40, pp.395-404, 1995.

T. Oguchi, A finite spectrum assignment for retarded nonlinear systems and its solvability condition, IJC, 80, pp.898-907, 2007.

T. Oguchi, A. Watanabe \& T. Nakamizo, Input-output linearization of retarded non-linear systems by using an extension of Lie derivative, IJC, 75, pp.582-590, 2002.

W. Respondek, A. Pogromsky \& H. Nijmeijer, Time scaling for observer design with linearizable error dynamics, Autom., pp.277-285, 2004

Y. Wang \& A. Lynch, A block triangular form for nonlinear observer design, IEEE TAC, pp.1803-1808, 2006

X-H Xia \& W-B. Gao, Nonlinear observer design by observer error linearization, SIAM J. Cont. Opt., 27, pp.199-216, 1989.

X. Xia , L.A. Marquez-Martinez, P. Zagalak \& C.H. Moog, Analysis of nonlinear time-delay systems using modules over non-commutative rings, Autom., 38, pp.1549-1555, 2002 